\title{
Living Donor Kidney Donation: Another Form of White Coat Effect
}

\author{
Stephanie S. DeLoach $^{a}$ Kevin E.C. Meyers ${ }^{b}$ Raymond R. Townsend ${ }^{c}$ \\ Departments of Medicine, a Thomas Jefferson University, ${ }^{\mathrm{b}}$ Children's Hospital of Philadelphia, and \\ 'University of Pennsylvania, Philadelphia, Pa., USA
}

\section{Key Words}

Ambulatory blood pressure monitoring $\cdot$ Blood pressure $\cdot$

Living donors $\cdot$ Hemodynamics $\cdot$ Transplantation

\begin{abstract}
Background/Aims: Living donor nephrectomy can be associated with increases in blood pressure several years following the procedure, but the best method to assess blood pressure during the living donor evaluation process is unclear. Methods: Living kidney donors underwent casual clinic and ambulatory blood pressure monitoring (ABPM) and measurement of central aortic pressures at baseline and 6 months following donor nephrectomy. Results: There was a significant decline in clinic systolic blood pressure (SBP; $p=0.001)$ and central aortic systolic pressure $(p=0.011)$ during the study period. However, average ABPM was unchanged and other measures of central arterial pressures and Augmentation Index were unchanged at 6 months compared to baseline. Conclusions: The remarkable differences between clinic SBP and ambulatory SBP prior to donation, and the disappearance of these differences 6 months later, suggest a substantial white coat effect on SBP associated with living kidney donor evaluation. Also, ABPM represents a better way to assess blood pressure prior to kidney donation.
\end{abstract}

Copyright $\odot 2011$ S. Karger AG, Basel

\section{Introduction}

The use of living kidney donors is one way to circumvent what is often several years of delay between dialysis initiation and kidney transplantation. There are concerns with using living donors, including an increased level of blood pressure in the future [1]; however, this is generally not observed until 5-10 years after donation. Currently, the hemodynamic evaluation of kidney donors is usually limited to clinic blood pressure levels, and sometimes ambulatory blood pressure monitoring (ABPM). ABPM is burdensome and expensive. However, there is recognition that clinic-based brachial-derived blood pressure levels fall short in their ability to represent the blood pressure when it is measured in the ambulatory setting. The same holds true for clinic-based brachial blood pressures to reflect pressure in the aorta [2], in which blood pressure levels are now accurately measured noninvasively with enhanced recognition of cardiovascular risk when compared with standard brachial blood pressures [3]. Therefore, clinic-based blood pressures do not appear to characterize fully future cardiovascular risk on several fronts [4]. Moreover, limited data suggests a high incidence of white coat hypertension during the kidney donation evaluation $[5,6]$. However, to our knowledge, no data details the persistence of white coat effect after kid-

Raymond R. Townsend, MD

University of Pennsylvania

3400 Spruce Street, 122 Founders Building

Philadelphia, PA 19104 (USA)

Tel. +1 215662 4630, E-Mail townsend@exchange.upenn.edu 
ney donation. Consequently, we hypothesized that the evaluation process for kidney donation may be attended by a substantial prevalence of white coat effect, even within those whose blood pressure is within the normal range, which may have attendant central aortic pressure changes detectable in the casual clinic setting. For the purpose of this study, we defined the 'white coat effect' as having significantly greater clinic compared to daytime blood pressures as recorded during 24-hour ABPM. For our study, we did not require that the clinic blood pressures be in the hypertensive range. We conducted this study to examine the effect of unilateral nephrectomy on clinicbased and central arterial pressures along with 24-hour ABPM before and 6 months following nephrectomy in living kidney donors.

\section{Subjects and Methods}

\section{Subjects}

Subjects included 17 living kidney donors, none of whom were taking antihypertensive medications. All subjects provided informed written consent and the study protocol was approved by the Institutional Review Board of the University of Pennsylvania.

\section{Clinical and Hemodynamic Measures}

Brachial blood pressures were recorded from the right arm in the seated position by the nursing staff in the Transplant Program using a Dinamap device (GE Healthcare, Milwaukee, Wisc., USA). Six-month follow-up blood pressures were performed by nursing personnel in the Clinical and Translational Research Center using a Datascope device (Datascope, Mahwah, N.J., USA) located adjacent to the Transplant Program offices. Hemodynamic measurements were performed shortly afterwards with the subject in the seated position after at least 5 min of rest. Central aortic systolic pressure, central pulse pressure, Augmentation Index and the time to wave reflection were obtained using the SphygmoCor system (AtCor Medical, West Ryde, Australia). Subjects were studied upon enrollment and 6 months following donor nephrectomy. Subjects also underwent 24-hour ABPM using the Spacelabs 90217 or 90207 monitors (Spacelabs Medical, Issaquah, Wash., USA) programmed to measure blood pressure in the nondominant arm at 20-min intervals during daytime and hourly during nighttime.

Renal function was determined from medical records data. Estimated glomerular filtration rate was calculated using the simplified modification of diet in renal disease equation [7].

\section{Statistics}

Descriptive statistics were used to characterize subject demographics and clinical parameters. A paired t test was used to evaluate change in hemodynamic and laboratory parameters within subjects over the study time points. To determine possible differences in the effect of location (clinic vs. ABPM) before and after donation we performed a repeated measure ANOVA. This procedure allows the determination of whether a response, in this case
Table 1. Clinical, hemodynamic and laboratory parameters of living kidney donors at baseline and 6 months after donor nephrectomy

\begin{tabular}{lccr}
\hline Parameter & $\begin{array}{l}\text { Baseline } \\
\text { (SD) }\end{array}$ & $\begin{array}{l}\text { 6 months } \\
\text { postnephrectomy } \\
(S D)\end{array}$ & \\
& & p & \\
\hline Age, years & $40.2 \pm 10.08$ & - & \\
Male (n = 9) & $53 \%$ & - & \\
Female (n = 8) & $47 \%$ & - & \\
BMI & $26.3 \pm 4.1$ & $26.0 \pm 4.2$ & 0.703 \\
Clinic SBP, mm Hg & $125 \pm 10$ & $115 \pm 8$ & 0.001 \\
Clinic DBP, mm Hg & $76 \pm 7$ & $74 \pm 6$ & 0.227 \\
Serum creatinine, mg/dl & $0.9 \pm 0.18$ & $1.3 \pm 0.24$ & $<0.001$ \\
eGFR, ml/min/1.73 m ${ }^{2}$ & $86 \pm 10.3$ & $60 \pm 11.7$ & $<0.001$ \\
Related, yes & $60 \%$ & - & \\
Average 24-hour, mm Hg & & & \\
$\quad$ SBP & $110 \pm 7$ & $111 \pm 8$ & 0.553 \\
$\quad$ DBP & $72 \pm 6$ & $72 \pm 7$ & 0.564 \\
$\quad$ Daytime SBP & $115 \pm 6$ & $116 \pm 10$ & 0.657 \\
$\quad$ Daytime DBP & $75 \pm 6$ & $76 \pm 7$ & 0.647 \\
$\quad$ Nighttime SBP & $98 \pm 9$ & $100 \pm 8$ & 0.415 \\
$\quad$ Nighttime DBP & $61 \pm 7$ & $63 \pm 6$ & 0.302 \\
\hline
\end{tabular}

the systolic blood pressure (SBP), was affected differently by the two 'factors' (i.e. the clinic compared with ambulatory setting). In addition, we performed a paired t test of the predonation SBP in the clinic compared with the predonation daytime SBP using the ABPM to test for white coat effect. Analyses were performed using STATA v11.0 (SAS Institute, Carey, N.C., USA).

\section{Results}

Characteristics of the study participants are presented in table 1 . A total of 17 donors had predonation and 6 months postdonor nephrectomy measurements completed. Subjects (16 Caucasian, 1 African-American) were between the ages of 29 and 59. Serum creatinine increased and estimated glomerular filtration rate decreased during the study period $(\mathrm{p}<0.001)$. Ambulatory and clinic blood pressure predonation and 6 months postdonor nephrectomy for each subject are presented in table 2 . The differences in clinic SBP from baseline to 6 months were significant ( $\mathrm{p}=0.001)$. The other blood pressure parameters in table 2 were not different at baseline compared to postdonation. The decline in clinic SBPs postnephrectomy compared to baseline is shown in figure 1. Paired $t$ testing showed a significant difference in predonation SBP in the clinic compared with the predonation daytime SBP on ABPM $(p<0.001)$. ANOVA testing showed a sig- 
Table 2. Clinic and 24-hour ABPM blood pressures at baseline and 6 months postnephrectomy for all study participants

\begin{tabular}{|c|c|c|c|c|c|c|c|c|}
\hline \multirow{2}{*}{$\begin{array}{l}\text { Subject } \\
\text { No. }\end{array}$} & \multicolumn{4}{|l|}{ Baseline } & \multicolumn{4}{|c|}{6 months postnephrectomy } \\
\hline & $\begin{array}{l}\text { clinic SBP } \\
\mathrm{mm} \mathrm{Hg}\end{array}$ & $\begin{array}{l}\text { ABPM } \\
\text { daytime SBP }\end{array}$ & $\begin{array}{l}\text { clinic } \\
\text { DBP }\end{array}$ & $\begin{array}{l}\text { ABPM } \\
\text { daytime DBP }\end{array}$ & $\begin{array}{l}\text { clinic } \\
\text { SBP }\end{array}$ & $\begin{array}{l}\text { ABPM } \\
\text { daytime SBP }\end{array}$ & $\begin{array}{l}\text { clinic } \\
\text { DBP }\end{array}$ & $\begin{array}{l}\text { ABPM } \\
\text { daytime DBP }\end{array}$ \\
\hline 1 & 128 & 109 & 80 & 60 & 122 & 94 & 72 & 57 \\
\hline 2 & 124 & 114 & 83 & 76 & 116 & 111 & 82 & 72 \\
\hline 3 & 114 & 108 & 80 & 67 & 102 & 112 & 66 & 65 \\
\hline 4 & 142 & 125 & 87 & 80 & 120 & 111 & 82 & 75 \\
\hline 5 & 120 & 116 & 80 & 73 & 110 & 115 & 80 & 76 \\
\hline 6 & 134 & 124 & 84 & 75 & 117 & 126 & 71 & 74 \\
\hline 7 & 133 & 118 & 65 & 71 & 105 & 108 & 70 & 68 \\
\hline 8 & 112 & 117 & 68 & 66 & 120 & 133 & 71 & 67 \\
\hline 9 & 118 & 114 & 74 & 69 & 107 & 119 & 72 & 75 \\
\hline 10 & 135 & 112 & 72 & 72 & 119 & 125 & 68 & 72 \\
\hline 11 & 133 & 118 & 82 & 74 & 110 & 119 & 70 & 74 \\
\hline 12 & 139 & 118 & 84 & 85 & 123 & 130 & 82 & 88 \\
\hline 13 & 127 & 108 & 63 & 71 & 133 & 116 & 76 & 72 \\
\hline 14 & 123 & 121 & 76 & 76 & 113 & 118 & 70 & 75 \\
\hline 15 & 113 & 122 & 74 & 71 & 121 & 118 & 73 & 74 \\
\hline 16 & 123 & 112 & 77 & 70 & 112 & 118 & 63 & 75 \\
\hline 17 & 108 & 108 & 68 & 60 & 108 & 105 & 68 & 65 \\
\hline Average & 125.1 & 115.5 & 76.3 & 71.5 & 115.2 & 116.4 & 72.7 & 72.0 \\
\hline SD & 10.0 & 5.6 & 7.2 & 6.3 & 7.9 & 9.5 & 5.8 & 6.6 \\
\hline
\end{tabular}

nificant interaction only for clinic SBP comparing baseline to postnephrectomy ( $\mathrm{F}$ ratio of 16.28; $\mathrm{p}=0.001$, posthoc testing using Huynh-Feldt epsilon).

Measures of wave reflection and central blood pressures are presented in table 3. Central aortic systolic pressure declined significantly following donor nephrectomy $(\mathrm{p}=0.011)$. There was a trend to lower central pulse pressure following donor nephrectomy, however, this was not statistically significant $(\mathrm{p}=0.096)$. The remaining parameters, Augmentation Index and time to first wave reflection were unchanged following donor nephrectomy.

\section{Discussion}

Our data show a substantial decrease limited to SBP comparing predonation to postnephrectomy values in our normotensive subjects undergoing living kidney donation. Eleven of our 17 subjects had more than a 10 $\mathrm{mm} \mathrm{Hg}$ fall in clinic SBP after 6 months. We also report that ABPM blood pressures were stable 6 months after donor nephrectomy. Given the discrepancy in SBP in the clinic versus ABPM in the predonation period the results

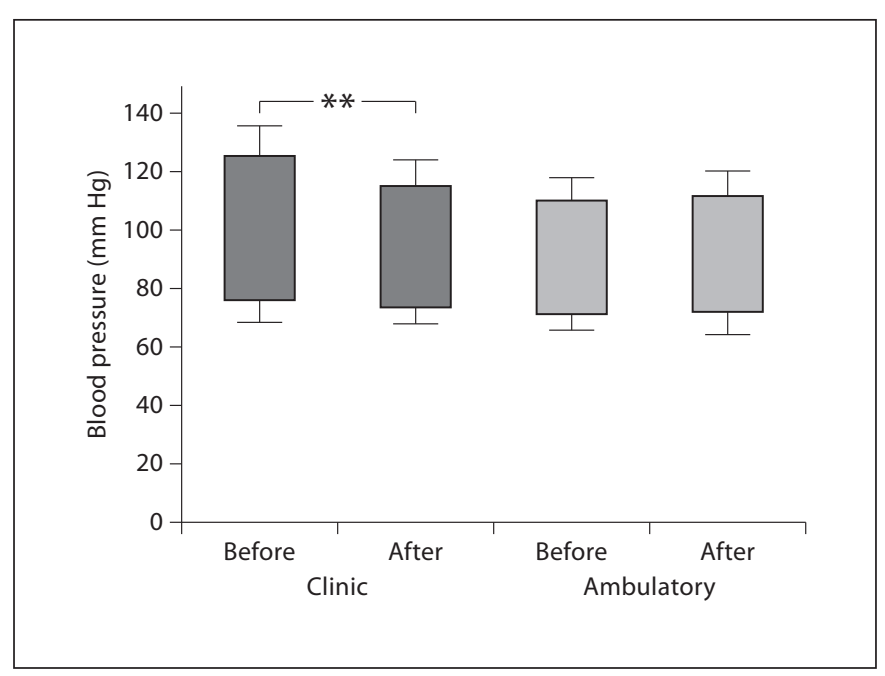

Fig. 1. On the left-hand side are floating bars (with SD error bars) showing clinic systolic and diastolic blood pressures before and 6 months following living kidney donation. On the right-hand side are floating bars (with SD error bars) showing mean systolic and diastolic ambulatory blood pressure before and 6 months following living kidney donation. ${ }^{* *} \mathrm{p}=0.001$. 
Table 3. Central hemodynamics before and following living kidney donation

\begin{tabular}{lccc}
\hline Parameter & Baseline (SD) & 6 months later (SD) & $\mathrm{p}$ \\
\hline Central aortic systolic pressure, mm Hg & $113 \pm 11$ & $103 \pm 7$ & 0.011 \\
Augmentation Index, \% & $19 \pm 14$ & $19 \pm 14$ & 0.887 \\
Central pulse pressure, mm Hg & $34 \pm 9$ & $30 \pm 7$ & 0.172 \\
Time to detection of pressure wave return, ms & $146 \pm 15$ & $144 \pm 15$ & 0.939 \\
\hline
\end{tabular}

are suggestive of a white coat effect as shown by the significant difference in predonation SBP in the clinic compared with the ambulatory systolic blood pressure values. Among the central blood pressure values only the central aortic systolic pressure fell significantly after donation. Since the central aortic systolic pressure value is related to the brachial systolic pressure used to calibrate the waveform, the decline in central pressures noted at 6 months reflects the decline in brachial SBP also noted at 6 months.

Blood pressures obtained from ABPM are likely a better reflection of true blood pressure compared to clinic blood pressure measures. A number of studies have noted the value of ABPM when clinic blood pressures are in the hypertensive range among prospective living kidney donors [6]. Such studies have noted that among potential donors who have elevated blood pressure during clinic evaluation, $30-60 \%$ of those subjects will be in the normotensive range when studied with ABPM $[5,6]$. Our study adds to this literature by observing that this white coat effect is not limited to just those with hypertensive clinic blood pressures.

In the short term, living kidney donation appears to have minimal effects on blood pressure. A study of the short-term effects of donor nephrectomy found stable blood pressures at 6-12 months postnephrectomy according to ABPM and clinic measures [8]. More recently, a prospective analysis of living donors also found stable blood pressures at 6 months postnephrectomy according to ABPM [9]. In our prospective analysis, we demonstrate similarly that early after-donor nephrectomy blood pressure is stable by ABPM. Moreover, we report that the white coat effect is no longer present at 6 months with respect to clinic blood pressure evaluation. We did not specifically study potential reasons for such a strong white coat effect among our subjects, but the stress of seeing several unfamiliar health care personnel, including physicians, and the general apprehension about the effects of surgery, etc. are likely contributors to this effect [10].
There is some evidence that arterial stiffness increases following donor nephrectomy. In a cross-sectional analysis pulse wave velocity was increased among living kidney donors, who were on average 9 years postdonation, compared to controls [11]. We included measures of central blood pressure in this study to determine if changes were evident in the early period postnephrectomy. Although we observed a significant decline in central SBPs from baseline to 6 months postdonor nephrectomy, this is likely due only to the changes in brachial blood pressure since the decline is identical in magnitude $(10 \mathrm{~mm} \mathrm{Hg})$. Other measures of central blood pressures did not change significantly in the study period.

Our study has some limitations. The number of subjects studied was modest, reflecting those we could recruit within the 1 year timeframe of funding for this study. Our subjects were nearly all Caucasian, thus, our data does not generalize to other ethnic groups. Finally, although care was taken to obtain clinic blood pressures in accordance with standard protocols, the personnel taking and recording the baseline blood pressures were not the same at baseline and 6 months, although in both instances they were trained nursing personnel and in both instances an oscillometric blood pressure device was used.

The short-term effects of donor nephrectomy upon central and ambulatory blood pressure support the assumption of low cardiovascular risk of the procedure for well-selected donors. ABPM showed less change after 6 months, unlike clinic SBP. Though more burdensome for prospective donors, ABPM provides, in our experience, a more accurate and reproducible estimate of blood pressure when compared with the clinic blood pressures during the donor evaluation process. In addition, we observed that the white coat effect was extinguished at 6 months after donation providing assurance about the cardiovascular safety of kidney donation. 


\section{Acknowledgements}

The project described was supported by NIH Grant No. UL1RR024134 from the National Center for Research Resources, NIH Grant DK-067390 and the Macy Foundation.

\section{Disclosure Statement}

The results presented in this paper have not been published previously in whole or in part. None of the authors have relevant disclosures or conflicts of interest for this work.

\section{References}

-1 Boudville N, Prasad GV, Knoll G, Muirhead N, Thiessen-Philbrook H, Yang RC, RosasArellano MP, Housawi A, Garg AX: Metaanalysis: risk for hypertension in living kidney donors. Ann Intern Med 2006;145:185196.

-2 McEniery CM, Yasmin, McDonnell B, Munnery M, Wallace SM, Rowe CV, Cockcroft JR, Wilkinson IB: Central pressure: variability and impact of cardiovascular risk factors: the Anglo-Cardiff Collaborative Trial II. Hypertension 2008;51:1476-1482.

$\checkmark 3$ Roman MJ, Devereux RB, Kizer JR, Okin PM, Lee ET, Wang W, Umans JG, Calhoun D, Howard BV: High central pulse pressure is independently associated with adverse cardiovascular outcome the strong heart study. J Am Coll Cardiol 2009;54:1730-1734.
4 Williams B, Lacy PS, Thom SM, Cruickshank K, Stanton A, Collier D, Hughes AD, Thurston H, O'Rourke M: Differential impact of blood pressure-lowering drugs on central aortic pressure and clinical outcomes: principal results of the Conduit Artery Function Evaluation (CAFE) study. Circulation 2006;113:1213-1225.

-5 Ommen ES, Schroppel B, Kim JY, Gaspard G, Akalin E, de BG, Sehgal V, Lipkowitz M, Murphy B: Routine use of ambulatory blood pressure monitoring in potential living kidney donors. Clin J Am Soc Nephrol 2007;2: 1030-1036.

$\checkmark 6$ Textor SC, Taler SJ, Larson TS, Prieto M, Griffin M, Gloor J, Nyberg S, Velosa J, Schwab T, Stegall M: Blood pressure evaluation among older living kidney donors. J Am Soc Nephrol 2003;14:2159-2167.

$>7$ Levey AS, Coresh J, Greene T, Stevens LA, Zhang YL, Hendriksen S, Kusek JW, Van Lente F: Using standardized serum creatinine values in the modification of diet in renal disease study equation for estimating glomerular filtration rate. Ann Intern Med 2006;145:247-254.
$>8$ Textor SC, Taler SJ, Driscoll N, Larson TS, Gloor J, Griffin M, Cosio F, Schwab T, Prieto M, Nyberg S, Ishitani M, Stegall M: Blood pressure and renal function after kidney donation from hypertensive living donors. Transplantation 2004;78:276-282.

-9 Prasad GV, Lipszyc D, Huang M, Nash MM, Rapi L: A prospective observational study of changes in renal function and cardiovascular risk following living kidney donation. Transplantation 2008;86:1315-1318.

10 Verdecchia P, Schillaci G, Borgioni C, Ciucci A, Zampi I, Gattobigio R, Sacchi N, Porcellati C: White coat hypertension and white coat effect: similarities and differences. Am J Hypertens 1995;8:790-798.

11 Bahous SA, Stephan A, Blacher J, Safar ME: Aortic stiffness, living donors, and renal transplantation. Hypertension 2006;47:216221. 